\title{
Corela
}

Cognition, représentation, langage

17-1 | 2019

Vol. $17, \mathrm{n}^{\circ} 1$

\section{Franglais : la marque de fabrique de la presse à scandale française}

Elodie Martin

\section{OpenEdition}

Journals

Édition électronique

URL : http://journals.openedition.org/corela/7943

DOI : $10.4000 /$ corela.7943

ISSN : $1638-573 \mathrm{X}$

Éditeur

Cercle linguistique du Centre et de l'Ouest - CerLICO

Référence électronique

Elodie Martin, «Franglais : la marque de fabrique de la presse à scandale française », Corela [En ligne], 17-1 | 2019, mis en ligne le 17 juin 2019, consulté le 27 juin 2019. URL : http:// journals.openedition.org/corela/7943; DOI : 10.4000/corela.7943

Ce document a été généré automatiquement le 27 juin 2019

\section{(c) (i) (2)(2)}

Corela - cognition, représentation, langage est mis à disposition selon les termes de la licence Creative Commons Attribution - Pas d'Utilisation Commerciale - Partage dans les Mêmes Conditions 4.0 International. 


\title{
Franglais : la marque de fabrique de la presse à scandale française
}

\author{
Elodie Martin
}

\section{Introduction}

1 La présente contribution propose de se pencher sur un genre journalistique renvoyant à un domaine de la presse écrite "de spécialité » : les tabloïdes. Caractérisé de "presse à scandale", "presse à sensation", et autres dénominations peu élogieuses telles que «presse poubelle», ce genre journalistique est dénigré (Bardelot 1999; Labarthe 2009; L'Obs 2011) car il ne traite pas de faits d'actualité dits sérieux, mais relaye des informations plus ou moins vraies sur la vie privée des célébrités, comme en témoigne Labarthe (2009) :

À la question à nouveau posée de savoir quelle est la première des qualités qu'il faut avoir pour écrire des articles people, deux chefs de rubrique people ont en effet ajouté : «aimer écrire sur rien », En profitant du phénomène d'éloignement des stars (sur Britney Spears, par exemple, qui ne lira jamais la presse suisse romande, « on peut dire tout et n'importe quoi ».

Outre la véracité des faits que la presse people relate, son utilité est également remise en question. Le contenu informatif de cette presse est restreint à ce que l'on nomme familièrement des « potins » ou des « ragots » (Delporte 2006). Certains journalistes people évoquent même "l'absence totale de contenu informatif ou d'intérêt public » que représentent les sujets abordés dans la presse à sensation (Labarthe 2009). Les tabloïdes sont ainsi jugés inutiles car les informations qu'ils relayent sont fantaisistes et superficielles. Selon quelques lectrices de presse à sensation (Bardelot 1999'1), les tabloïdes sont amusants et distrayants mais ne constituent pas une lecture sérieuse ou utile. Du point de vue de certains journalistes dont Labarthe se fait l'écho (2009), la presse people est jugée peu sérieuse voire dégoûtante et, par conséquent, peu respectables. Pour résumer, les non professionnels du milieu journalistiques (Bardelot 1999) ainsi que les professionnels (Labarthe 2009) déprécient et décrédibilisent ce type de presse. 
3 Ainsi, si le fond est critiqué, la forme l'est étonnement beaucoup moins. La presse à sensation se distingue considérablement des autres types de presse via le lexique singulier dont elle fait usage. Elle alterne le français et l'anglais. Cette pratique, communément nommée " franglais " ${ }^{2}$, doit son nom à Maurice Rat (1959) (Bogaards 2008 : 13) et sa popularisation à Étiemble via son ouvrage Parlez-vous franglais? (1973). De nombreux non spécialistes et spécialistes (Allaoua 1992 ; Ducas 2014 ; Bruley 2018), l'un des plus célèbres étant Étiemble, décrient l'insertion de mots anglais en français. Ainsi entend-on parler, entre autres, de "gangrène » (Allaoua 1992: 84), d' «anglicisation du français », de «menace », d' " épidémie » ou encore de « contamination » pour reprendre les termes d'Étiemble (1973). La presse people pourrait alors également être critiquée pour ses choix linguistiques combinant le français et l'anglais, tout comme le sont les locuteur.rice's alternant ces deux langues dans leurs discours ${ }^{3}$. Cette pratique langagière a mauvaise réputation car elle essentiellement perçue comme le résultat d'un trouble ou d'une déficience chez le·la locuteurrice. Haugen (1977 : 94) énumère trois déficiences ou troubles qui, du point de vue des monolingues, mènent les locuteurrrice's maitrisant deux langues à les alterner ${ }^{4}$ : un manque de moral, une déficience intellectuelle, un trouble social ${ }^{5}$. Ainsi, en prenant appui sur les travaux d'Haugen, nous affirmons que les locuteur.rice's n'ayant pas recours au franglais perçoivent cette pratique langagière comme un comportement immoral laissant transparaître une forme de paresse liée au fait que les locuteurrice's ne tentent pas d'éviter d'alterner, au sein d'un même énoncé, les deux langues qu'ilselles maîtrisent. La potentielle déficience intellectuelle que les locuteur-rice's monolingues pensent percevoir via l'utilisation du franglais s'apparente à une faiblesse lexicale et traduit une maîtrise approximative du français ainsi que de l'anglais (Hoffman 1991: 109). En effet, concernant le français, les auditeurrice's pourront, par exemple, se demander pourquoi lela locuteurrice ne formule pas entièrement la phrase en français : le vocabulaire lui fait-il défaut (Rowlett $2006: 425)$ ? Tente-t-ileelle de dissimuler des lacunes en alternant le français et l'anglais ? À l'inverse, en ce qui concerne l'anglais, les auditeurrice's pourront s'interroger sur la réelle capacité du locuteur ou de la locutrice à s'exprimer en anglais, à se faire comprendre, et à interagir avec des anglophones ou, au moins, avec des locuteur.ricess ayant une bonne maitrise de l'anglais. Enfin, selon les locuteur.rice's n'ayant pas recours à cette pratique, l'utilisation du franglais, renvoie à une forme de snobisme et d'arrogance (Rowlett 2006 : 425) ainsi qu'à une façon pour lela locuteurrice francophone de chercher à épater son auditoire direct et/ou indirect ${ }^{6}$ en insérant des mots anglais dans son discours.

4 Pour résumer, les locuteurrice's ne recourant pas au franglais remettent en question la moralité des locuteur.rice's alternant le français et l'anglais, leurs aptitudes langagières, ainsi que leur connaissance des codes sociaux. Quant à la presse à scandale, ses lecteurrrice's et les journalistes people ou d'autres types de presse la dénigrent car les contenus qu'elle propose sont dits racoleurs et trop légers, et ses journalistes peu professionnels (Labarthe 2009). Dans le but d'introduire au mieux la relation entre les tabloïdes français et le franglais, et de mettre en exergue leur point commun, il nous a semblé primordial de débuter cet article en abordant la notion de "perception ", et de mentionner que la manière dont les professionnels et non professionnels perçoivent la presse à scandale coïncide avec la perception générale que les locuteur.rice's monolingues ont du franglais. En effet, tout comme la presse à scandale, le franglais est connoté négativement. 
5 Notre étude vise à présenter les principales raisons qui, selon nous, motivent considérablement l'insertion de mots anglais dans les tabloïdes français. Nous développerons les notions clés suivantes : l' «économie du langage » (Martinet 1955) et l' « identité de groupe » (Brown et Levinson 1987). Au préalable, nous aborderons, dans la première partie de l'article, les spécificités du franglais dans la presse people, à savoir l'aspect codé et ciblé de ce lexique ainsi que la notion de « quantité » à laquelle il renvoie car, comme le mentionne Rowlett (2006: 425) dans sa définition du franglais, les locuteur-rice's ayant recours au franglais insèrent de nombreux mots d'origine étrangère dans leur discours :

[...] Franglais refers to the phenomenon whereby native English or French speakers pepper their speech with lexis from the other language for humorous effect, to show off, or because of gaps in their native lexis ${ }^{7}$.

6 Concernant l'aspect codé et ciblé des mots anglais insérés dans les tabloïdes français, nous expliquerons pourquoi l'usage d'un certain type de mots anglais est privilégié par rapport à celui leurs équivalents français. Puis, nous traiterons de l'aspect quantitatif du franglais via la multitude d'expressions ou de mots anglais insérés dans les tabloïdes français.

7 Notre étude vise par conséquent à mettre en exergue de quelles manières le franglais se matérialise dans les tabloïdes français et pour quelles raisons les journalistes, rédacteurs en chef, et autres spécialistes des journaux à sensation font usage du franglais dans leurs magazines. Bien que la presse people soit un objet d'étude pour plusieurs chercheurs (Balle 1998 ; Marion 1999 ; Rais 2005 ; etc.), l'étude de ce genre journalistique et de son lexique singulier, dans une perspective sociolinguistique telle que celle que nous proposons, demeure un phénomène encore peu traité scientifiquement. De plus, ce sujet d'étude nous semble original et pertinent car il est surprenant que deux éléments ayant aussi mauvaise réputation se combinent et soient à l'origine d'un marché si prospère. L'usage du franglais fait, selon nous, partie des codes de la presse people française. Deux chefs de la rubrique people, dont Labarthe (2009) rapporte les propos, précisent que « savoir l'anglais » est l'une des compétences essentielles dans ce domaine. Ils expliquent que leurs connaissances en anglais leur permettent de :

[...] Repiquer, traduire et adapter en français des articles lus sur les sites des journaux («le Sun, le Daily Mail, [...], le Mirror, le News of the World, le People américain, le US Weekly... ») ou sur des sites Internet d'information anglo-saxonne dédiés aux stars (www.people.com, pour n'en citer qu'un).

8 Nous pouvons considérer que cette pratique a ainsi mené les journalistes people français à conserver des lexèmes ${ }^{8}$ anglais dans la presse à scandale française. Ceci témoigne de la tradition discursive caractéristique de la presse people française. Loiseau (2013) définit la notion de « tradition discursive » comme suit :

La notion de tradition discursive insiste sur les relations de répétition et d'imitation entre les textes : les textes sont d'abord de larges répétitions et imitations de textes antérieurs. Schlieben-Lange (1996) parle de «la répétition, la récurrence, le riche réservoir où puisent les sujets producteurs de textes"; "la répétition, la récurrence d'un stock de formules et des passages tout faits »; Koch (1997) de " comportement communicatif typique et reproductible»; Aschenberg (2003) souligne qu'il n'y a pas de discours qui ne « [recycle] des modèles généraux » [...].

Il y a ainsi « imitation » de la part des magazines people français qui s'inspirent des textes anglo-saxons et "répétition» des mêmes lexèmes anglais dans les divers tabloïdes français. 
10 En vue de montrer de quelles manières le franglais se matérialise dans la presse à sensation française, nous avons sélectionné les magazines de presse à scandale français Closer, Gala, Grazia, Paris Match, Public, Purepeople, et Voici. Afin de constituer notre corpus, nous proposons d'analyser le lexique teinté d'anglais propre aux articles people des rubriques "scoop ", " actu », et « news », aux gros titres, ainsi qu'aux titres d'articles et de séries de photos des versions en ligne des magazines précités. Notre sélection de gros titres, titres, et articles se limite aux années 2017 et 2018. Nous avons consulté ces références médiatiques à titre informatif, dans un premier temps, c'est-à-dire dans un cadre non-professionnel. Puis, leur contenu lexical nous a interpelé au point de nous interroger sur le franglais dans la presse à scandale et de consacrer une analyse scientifique à ce thème.

11 La première partie de cette contribution s'attachera à mettre en exergue quelques-unes des caractéristiques $\mathrm{du}$ franglais dont nous constatons la présence dans les tabloïdes français : son lexique anglais codé, ciblé, et conséquent.

\section{Les spécificités du franglais dans la presse à scandale française}

12 Dans la presse à sensation française, le franglais se matérialise par l'insertion de mots anglais dans des énoncés rédigés majoritairement en français, pour un public de francophones. À travers cette première partie, nous mettrons en lumière, dans un premier temps, la manière dont l'utilisation de l'anglais s'organise dans la presse à scandale française. Puis, dans un second temps, nous développerons l'une des notions clés devant être prise en compte dans l'étude du franglais : la « quantité ».

\subsection{Un lexique anglais codé et ciblé}

13 Dans le cadre des tabloïdes français, l'utilisation du franglais se traduit par le fait de privilégier l'usage d'un certain type de mots anglais plutôt que celui de leurs équivalents français.

14 Par exemple, il y a quelques mois, le magazine français Closer titrait: "Sam Asghari métamorphosé : le boyfriend ultra-sexy de Britney Spears ne ressemblait pas DU TOUT à ça » (Vincent, Closer.fr 2018). Dans cet exemple, le substantif anglais boyfriend ${ }^{9}$ remplace l'équivalent français « petit ami ». En parcourant les tabloïdes français, nous notons que ce mot est souvent préféré à son équivalent français, comme le montrent les exemples suivants : « Réunis à Las Vegas le temps d'une soirée, Céline Dion et son supposé nouveau boyfriend Pepe Munoz ont assisté ensemble à un spectacle érotique donné par le Cirque du Soleil » (El Himani, Gala.fr 2017) ; « Ariana Grande : déjà un nouveau boyfriend?» (Public.fr 2018) ; « Amber Heard : découvrez son nouveau boyfriend ! (Public.fr 2018).

15 Par opposition à boyfriend, nous remarquons également l'emploi de girlfriend, plutôt que "petite amie", dans la presse à scandale française. Afin d'illustrer cette affirmation, considérons les exemples suivants : «Et si M. Pokora a annoncé se « retirer quelques mois des reseaux sociaux» le 15 janvier dernier, il y avait fort à parier que sa girlfriend l'exposerait sur Instagram» (Purepeople.fr 2018); "Drake n'est plus célibataire! Découvrez le visage de sa nouvelle girlfriend » (Sall, Public.fr 2018) ; « Ian Somerhalder et 
sa girlfriend Nina Dobrev, très glamour, arrivent à l'aéroport de LAX pour se rendre à Atlanta » (Purepeople.fr 2018).

D'autres lexèmes anglais tels que l'adjectif «topless ", ayant pour équivalent français "seins nus ", sont aussi préférés dans la presse à sensation française, à l'image des exemples suivants : «Zazie topless, la photo osée dont TOUT le monde parle!» (JilotGuérand, Public.fr 2018); «Alizée topless à la plage... attention c'est chaud!» (JilotGuérand, Public.fr 2018) ; «Torride, Bella Hadid pose topless en couverture de Vogue » (ParisMatch.com 2018).

Nous remarquons alors que le vocabulaire anglais dont les journalistes de presse à scandale font usage est codé, en ce sens que les lexèmes étrangers insérés dans les énoncés français ne sont pas choisis au hasard. Au contraire, les journalistes mettent en évidence l'information principale, c'est-à-dire la nouvelle à communiquer, en utilisant l'anglais. Ainsi, dans les exemples précités, les informations capitales des énoncés sont les suivantes : $\mathrm{X}$ a un nouveau boyfriend ou une nouvelle girlfriend, et $\mathrm{Y}$ était topless sur la plage. Par « information principale » nous entendons « information que les lecteur.rice's viennent chercher dans ce type de presse ", ce qui renvoie à l'aspect ciblé du lexique propre aux tabloïdes français. En effet, nous considérons que boyfriend, girlfriend, et "topless» font partie du lexique singulier de la presse à scandale car les amours des célébrités, tout comme leur nudité, sont les sujets phares dont les lecteurrice's sont particulièrement friande-s. Ce qui intéresse les lecteur.rice's et que les journalistes privilégient renvoie essentiellement à l'intimité des personnalités célèbres. La presse à sensation fait de son fond de commerce l'intimité des célébrités qu'elle rend extime. Tisseron (2001: 52) « [...] propose d'appeler « extimité » le mouvement qui pousse chacun à mettre en avant une partie de sa vie intime, autant physique que psychique » (2001:52). La notion d' " extimité » (Tisseron 2001, 2011 ; Tournier 2002 ; Lacan 2006) telle qu'elle est définie par Tisseron s'applique tout à fait à la presse à scandale, excepté que cette extimité est forcée car l'intimité des personnalités est, la plupart du temps, dévoilée dans les magazines sans leur consentement préalable, comme l'explique Lits (2009) dans la citation suivante :

Le lecteur est invité à pénétrer, quasiment par effraction, dans la chambre à coucher de ses vedettes préférées, pour les surprendre dans leur intimité. En atteste la condamnation de certains magazines, sur plainte des vedettes photographiées à leur insu, ce qui permet d'authentifier cette intrusion voyeuriste, de la rendre encore plus visible. Les amours des people n'existent que pour être dérobées et exhibées aux yeux de tous.

18 Dans un esprit quelque peu voyeur (Jouve 1992 ; Lits 2009), la mise à nu des people, au sens propre comme au figuré, aiguise la curiosité des lecteurrice's et les fascine (Lits 2009). La frontière entre vie privée et vie publique est largement franchie dans la presse people. Ce qui est privé devient public. Le lectorat pense ainsi tout connaître des stars qui voient leur intimité dévoilée dans les magazines. Schickel (1985) nomme intimate strangers, " étrangers intimes » en français, ces célébrités dont les anonymes pensent tout connaitre car leur intimité est dévoilée. Au niveau lexical, l'accent est mis sur l'alternance du français et de l'anglais, l'anglais servant à transmettre les informations en lien avec l'extimité des célébrités que le lectorat recherche dans ce type de presse. Jouve (1992) explique que «toute lecture est fondée sur la curiosité». Dans le cas de la presse à scandale, l'extimité des célébrités qui renvoie, entre autres, aux récits de leurs vies sentimentales (boyfriend, girlfriend) ainsi qu'aux photographies de leurs corps dénudés (topless), motive la lecture des lecteurrrice's de tabloïdes curieux'ses d'être informée's des 
nouveaux couples ainsi que des ruptures, et désireux:ses de découvrir l'intimité physique des stars.

La sous-partie suivante sera axée sur la notion de " quantité » (Rowlett 2006: 425). Au vu du nombre important de lexèmes anglais que nous avons recensés dans les tabloïdes français sélectionnés, il nous semble nécessaire d'intégrer une démonstration quantitative à notre réflexion.

\subsection{Un lexique anglais abondant}

En prenant appui sur la définition du franglais de Rowlett (2006:425) et en l'adaptant à notre cas d'étude, nous pouvons affirmer que les locuteurrice's francophones ayant recours au franglais ont tendance à insérer un certain nombre de mots ou groupes de mots anglais dans leur discours. Ilseelles ne parsèment pas simplement leur discours de quelques lexèmes anglais mais alternent fréquemment les deux langues. L'analyse du franglais implique donc de prendre en compte son aspect quantitatif.

21 La presse people française regorge également d'expressions et de mots anglais, contrairement aux autres types de presse ${ }^{10}$. Le franglais est abondamment présent dans les tabloïdes français. Nous constatons sa présence dans les titres et extraits d'articles, comme illustré par les exemples précédents, ainsi que dans les gros titres, comme en témoignent les cinq captures d'écran regroupées en Annexe 1. Elles représentent les premiers résultats qu'une recherche sur Internet des magazines français Gala, Paris Match, Public, Purepeople, et Voici génère. Cette recherche a été effectuée après avoir pris soin d'effacer l'historique de navigation pour que cette recherche ne soit pas conditionnée par les précédentes et de sélectionner la langue française uniquement dans les préférences de navigation. Sur ces captures d'écran, nous avons souligné les lexèmes anglais en rouge afin de les mettre en évidence. Nous dénombrons un total de 44 expressions et mots anglais insérés. La récurrence des lexèmes tels que "people", "stars", et "news people", est patente. Ces captures d'écran illustrent que les tabloïdes français sont empreints d'anglais. Ainsi, nous constatons que la presse à sensation présente les mêmes caractéristiques du franglais que les locuteurrice's ayant recours à cette pratique langagière. Les deux langues sont alternées des sous-titres des magazines aux titres des diverses rubriques, en passant par les descriptifs des magazines et de leurs rubriques.

Le sémantisme du lexique utilisé dans la presse à scandale semble codé car l'information principale ${ }^{11}$ est en anglais, mais il est aussi assez restreint et, par conséquent, ciblé. En effet, les champs lexicaux qui se dégagent des exemples fournis via les captures d'écran, les titres, et les extraits d'articles de la précédente section correspondent aux sujets types que la presse à sensation cible car ils font rêver le lectorat, l'intrigue, et, par conséquent, font vendre (Labarthe 2009; Lits 2009). Ainsi, ses domaines de prédilection sont les suivants : les célébrités, leur actualité professionnelle, qui englobe les projets artistiques actuels ou à venir (films, séries, albums, tournées, etc.), leurs amours, leur nudité (Lits 2009), mais également la royauté et, plus précisément, les naissances ainsi que les mariages, qui font, selon nous, particulièrement rêver le lectorat de presse people. Les mariages royaux et les naissances royales captivent et fascinent (Lits 2009) les lecteur.rice-s, voire les émerveillent, car ils font écho aux récits, contes, et légendes. De plus, selon Lits (2009), les journaux people traitent ce type d'événements princiers en suivant les modèles des contes et légendes, ce qui renforce l'analogie entre la réalité et la fiction. 
aux divers exemples apportés au cours de cet article ${ }^{12}$, nous constatons que les lexèmes renvoyant aux célébrités sont «stars » et «people». Les journalistes font référence à l'actualité des célébrités via les mots et expressions " news », " news people ", "news de stars», "news TV», topnews, livenews, «scoop», et «buzz». La vie sentimentale des personnalités célèbres est racontée et exposée par le biais des substantifs boyfriend et girlfriend. Nous notons également l'emploi de l'expression love story, comme en témoignent les phrases d'exemples suivantes : « Pamela Anderson et Adil Rami : alerte à la love story » (Purepeople.fr 2018); «Vanessa Paradis pousse un coup de gueule sur sa love story avec Samuel Benchetrit: «Je n'ai pas à le partager » ( (Faure, Closer.fr 2018). Le principal lexème renvoyant à la nudité des stars est « topless ». Ce qui a trait à la royauté est généralement répertorié dans la catégorie Royal blog de la presse à scandale qui comporte des informations sur les naissances (royal baby), comme en témoignent les titres d'articles suivants renvoyant à la famille royale britannique: «Pourquoi le royal baby s'appelle-t-il Louis, Arthur, Charles?» (Mathieu, ParisMatch.com 2018); «Le royal baby 3 le fils de Kate Middleton et William rapporte déjà gros » (Rouyer, Gala.fr 2018), mais également sur les mariages (royal wedding) : "Royal wedding: Meghan Markle et le Prince Harry faits duc et duchesse de Sussex » (Santrot, Grazia.fr 2018) ; « Le royal wedding a 2 mois : 30 photos pour revivre le conte de fées de Meghan et Harry!» (Public.fr 2018).

Le franglais est alors le marqueur lexical par excellence de la presse à scandale française. Nous le constatons via l'insertion de lexèmes anglais dans des énoncés dont la langue dominante est le français, via la récurrence et la fréquence de ces lexèmes dans des énoncés plus ou moins courts tels que les gros titres, les titres, et les phrases extraites d'articles, ainsi que via la récurrence et la fréquence des thématiques abordées.

Dans la partie suivante, il s'agira de déterminer les raisons de l'utilisation d'un lexique si particulier car teinté d'anglais.

\section{Les raisons du franglais dans la presse à scandale française}

Nous souhaitons centrer cette seconde partie sur deux des principales motivations que nous attribuons à l'utilisation du franglais dans les tabloïdes français : l'économie du langage et l'identité de groupe.

\subsection{L'économie du langage}

L'une des principales raisons justifiant, selon nous, la présence du franglais dans les tabloïdes français est liée aux spécificités de la presse écrite en général, à savoir la nécessité pour les journalistes d'attirer l'attention des lecteurrrice-s. Insérer des mots anglais dans les magazines people français est alors un moyen pour les journalistes de capter l'attention des lecteurrrice's francophones ${ }^{13}$. Cette stratégie linguistique nous renvoie à la notion de "saillance » qui se trouve être, dans notre cas d'étude, lexicale et visuelle, conformément à la définition suivante fournie par Landragin (2006) :

Est saillant ce qui vient en premier à l'esprit, ce qui capte l'attention. Cette propriété, parfois appelée prosexigène (obtrusive en anglais), s'applique aux entités $\mathrm{du}$ discours via les caractéristiques lexicales, syntaxiques et sémantiques du discours, auxquelles il faut ajouter les caractéristiques phonétiques et prosodiques 
dans le cas du discours oral et les caractéristiques visuelles dans le cas du discours écrit. La notion de saillance (salience ou saliency en anglais) est ainsi liée à l'émergence d'une figure sur un fond, que cette émergence soit motivée par des aspects physiques liés à la perception de la parole ou du texte écrit, ou par des aspects plus sémantiques voire cognitifs liés à la compréhension du langage. C'est pourquoi on peut distinguer la saillance physique de la saillance cognitive, de manière à mieux rendre compte de phénomènes qui peuvent jouer dans le même sens ou dans des sens opposés. concis, en particulier dans les gros titres des magazines ainsi que dans les titres d'articles. L'objectif est de parvenir à transmettre une information par le biais d'un énoncé court. Dans de nombreux cas que nous détaillons ci-dessous, l'utilisation des lexèmes anglais précédemment analysés, par opposition à celle leurs équivalents français, permet d'aller droit au but en utilisant un minimum de mots ou de syllabes. Ce phénomène linguistique est appelé l' « économie du langage » (Martinet 1955), ou encore le "principe du moindre effort », et est défini comme suit par Martinet (1980 : 176-177) :

L'évolution linguistique peut être conçue comme régie par l'antinomie permanente entre les besoins communicatifs de l'homme et sa tendance à réduire au minimum son activité mentale et physique. Ici, comme ailleurs, le comportement humain est soumis à la loi du moindre effort selon laquelle l'homme ne se dépense que dans la mesure où il peut ainsi atteindre aux buts qu'il s'est fixés.

Ainsi, dans notre cas d'étude, le concept d'économie du langage se traduit par l'insertion de lexèmes anglais dans les gros titres, les titres, mais également dans les articles de presse française car ils donnent l'opportunité aux journalistes de transmettre une idée identique à celle que les équivalents français auraient transmise tout en réduisant le nombre de mots ou de syllabes. Concernant l'économie du langage liée à la praticité des emprunts, Dunbar (2017) explique ce qui suit :

Ils servent [...] à être plus concis dans la mesure où l'équivalent d'une expression peut être plus court dans une autre langue. Prenons le cas de l'emprunt en anglais de l'expression française "déjà vu ", il est clair qu'emprunter des mots étrangers peut être très pratique. Au lieu de dire «This feels like something that's happened to me before », en anglais, il est parfaitement acceptable de simplement dire «It's like déjà vu ».

Par conséquent, utiliser boyfriend, girlfriend, "topless", "news people», topnews, et livenews, plutôt que leurs équivalents français respectifs "petit ami/petit copain", " petite amie/petite copine », « seins nus », « nouvelles de célébrités/nouvelles de stars", " informations/infos à la une », et "informations/infos en direct», est un moyen de réduire le nombre de mots. De la même manière, selon nous, l'utilisation de "stars " (une syllabe) et "people» (deux syllabes), "news» (une syllabe), "news de stars» (trois syllabes), «news TV » (trois syllabes), «scoop» (une syllabe), «buzz» (une syllabe), et love story (trois syllabes) permet de réduire le nombre de syllabes par rapport à celui de leurs équivalents français "célébrités " (quatre syllabes) et "personnalités célèbres " (sept syllabes), «nouvelles/actualité» (deux syllabes/cinq syllabes), "nouvelles de célébrités/nouvelles de stars " (sept syllabes/quatre syllabes), "actualité (à la) télé " (sept (neuf) syllabes), "exclusivité » (cinq syllabes) voire "exclu» (deux syllabes), « événement » (quatre syllabes), et « histoire d'amour » (quatre syllabes).

Pour résumer, selon nous, l'économie du langage est l'une des raisons expliquant la présence du franglais dans la presse à sensation française. Cependant, recourir à l'identité 
de groupe comme stratégie commerciale nous semble être une raison supplémentaire justifiant l'alternance du français et de l'anglais dans cette presse.

\subsection{L'identité de groupe} informations relayées par les tabloïdes participent de l'identité de groupe. Concernant la forme, la langue en elle-même n'a, selon nous, pas réellement d'importance. En effet, ce n'est pas l'anglais qui crée un phénomène d'identification chez les lecteurrice-s, mais plutôt le style informel qu'engendre l'alternance du français et de l'anglais, dans ce type de presse, et que l'on ne retrouve dans aucune autre presse. Nous avons tendance à percevoir l'alternance de deux langues, du français et de l'anglais concernant notre cas d'étude, comme une pratique langagière synonyme de familiarité. En effet, nous considérons que l'usage du franglais est à éviter dans toute situation nécessitant une certaine formalité car il s'apparenterait à une forme d'impolitesse (Brown et Levinson 1987; Culpeper 2011). Ainsi, dans une lettre de motivation, ou lors d'un entretien d'embauche face à un potentiel patron, par exemple, alterner deux langues est à proscrire. Dans la presse people, le franglais traduit également une certaine familiarité, un manque de formalité, voire une attitude amicale face au lectorat, ce qui laisse, selon nous, plus aisément la place à l'identification aux célébrités. Il convient également d'ajouter que ce genre discursif caractéristique de la presse people répond aux attentes du lectorat, assimilé, dans notre cas d'étude, à une communauté. Comme précisé par les témoins dont Bardelot (1999) rapporte les propos, lire ce type de presse a vocation à détendre léla lecteurrrice. Le ton informel que cette presse emploie participe donc de la relaxation du 
lectorat. La notion de "communauté discursive" (Swales 1990) nous permet ici de renvoyer d'une part au caractère non formel du discours de la presse people qui constitue un genre discursif qui lui est propre et qui répond aux attentes du lectorat, et d'autre part à son lexique spécifique combinant le français et l'anglais.

Concernant le fond, la presse à scandale a une fonction identitaire, comme l'explique Lits (2009) dans la citation suivante :

Cette identification peut autant se faire par identité des trajets présentés avec ceux $\mathrm{du}$ lectorat principal de ces publications (la vedette qui est partie de rien, à la Johnny, ou les exemples récents de Star Academy et des programmes de télé-réalité, en suivant les parcours de Loana ou Jenifer) que par proximité affective (elle a souffert comme nous; malgré son argent, la vedette connaît des malheurs; nous sommes tous égaux devant la mort, etc.). L'important est de jouer sur la double face de la star, à la fois être idéal auquel le lecteur peut s'identifier, et personne semblable à lui. Comme le rappelle Jean-Pierre Esquenazi (2003), «il leur faut montrer à la fois qu'elles sont des individus comme les autres et des personnes extraordinaires, qu'elles sont à la fois communes et héroïques » (p. 62).

Morin (1984 : 381) mentionne également la double condition des célébrités qu'il décrit comme des êtres à la fois humains et surhumains :

Les stars de l'époque ont une double nature, humaine et surhumaine. Ce ne sont plus les déesses inaccessibles du muet; par mille aspects familiers, elles ressemblent au commun des mortels et se proposent comme héros-modèles de la civilisation individualiste-hédoniste ; mais elles vivent en même temps à un niveau supérieur d'intensité et de qualité, elles sont une substance divine qui appelle l'adoration, elles incarnent une liberté fabuleuse que les mortels ne peuvent atteindre. Elles se trouvent au carrefour entre la vie idéale et la vie réelle et constituent la grande plaque tournante entre le réel et l'imaginaire.

37 Ainsi, les lecteur-rice's des tabloïdes peuvent, selon nous, plus facilement s'identifier aux aventures sentimentales des célébrités, par exemple, qui ne sont, en définitive, pas si différents des leurs, qu'à des sujets plus sérieux et plus graves tels que les manigances politiques, les guerres, le terrorisme, etc. relatés dans d'autres types de presse. Nous considérons que ces sujets ont une fonction identitaire bien moindre, voire quasiment inexistante, car ils sont extrêmement déroutants. L'identification aux célébrités est d'autant plus aisée pour les lecteur.rice's qu'elle «permet de vivre par procuration l'existence qu'on rêve de connaître " (libido dominandi) (Lits 2009), par opposition aux sujets plus dramatiques énumérés ci-dessus. La presse à scandale a pour objectif de faire rêver ses lecteur.rice's tout en leur faisant croire que les célébrités leur ressemblent, ont les mêmes joies, les mêmes problèmes, et les mêmes préoccupations ( $\mathrm{X}$ emménage avec $\mathrm{Y}$, $X$ se sépare de $Y$ et se met en couple avec $Z$, X et $Y$ attendent un enfant, etc.), afin de créer un sentiment d'identification. Ce sentiment de projection-identification (Morin 2008) fait écho à la définition de la notion d' « identification » donnée par Lalande (1968) :

Processus par lequel un individu $A$ transporte sur un autre $B$, d'une manière continue plus ou moins durable, les sentiments qu'on éprouve ordinairement pour soi, au point de confondre ce qui arrive à $\mathrm{B}$ avec ce qui lui arrive à lui-même.

Concernant les récits de vie ou d'événements qui, aux yeux des personnes anonymes, paraissent exceptionnels, singuliers, voire irréels tels que les destinées princières, par exemple, l'identification du lectorat aux people est moins évidente. Les dimensions du rêve et du conte de fée (Bellemin-Noël 1983) entrent alors en jeu. Elles donnent aux situations auxquelles le lectorat peut s'identifier plus facilement un sentiment d'identification plus intense. Nous considérons que c'est cette ambivalence, à laquelle 
Esquenazi (2003 : 62) et Morin (1984) font référence, qui aiguise la curiosité du lectorat et le fascine. En d'autres termes, le lectorat n'a pas besoin de partager tous les traits distinctifs d'une célébrité pour parvenir à s'identifier à elle, mais il se sent d'autant plus proche de la star lorsqu'il découvre leurs caractéristiques communes.

Bien que dans certaines situations les people semblent être inaccessibles, la plongée dans leur vie privée que les tabloïdes offrent créée, du point de vue du lectorat, l'illusion d'une intimité avec les célébrités, comme l'explique Stoetzel, cité par Balle (1994 : 209) :

[...] les anecdotes ou récits concernant la vie privée des célébrités du monde du spectacle, créent avec le lecteur une forme d'intimité. Il a l'impression de mieux connaître et de côtoyer des personnages qui lui sont totalement inaccessibles dans sa vie de tous les jours.

Par ailleurs, en dévoilant l'intimité des personnalités du monde entier, ceci faisant écho à la notion d' " extimité " précédemment abordée, les journalistes donnent l'impression aux lecteur.rice's d'être des privilégiée's à qui ilselles font des confidences. L'idée de privilège renforce davantage le concept d'identité de groupe que suggère, selon nous, le franglais dans la presse à sensation. En effet, la notion d' «identité de groupe » implique nécessairement que les individus et locuteur-rice-s étrangers aux spécificités du groupe en question en soient exclus. À travers ce type de confidences sur les personnalités célèbres, les journalistes souhaitent faire croire aux lecteurrice's qu'ilselles font partie d'un groupe de privilégiée's informé des moindres secrets des célébrités dont sont exclues les personnes ne lisant pas ce type de presse.

41 Ainsi, la légèreté de cette presse, dans le fond comme dans la forme, est une stratégie commerciale qui vise à créer une certaine intimité avec les lecteurrrice's et à les faire s'identifier plus aisément aux changements positifs et négatifs qui surviennent dans la vie des célébrités, afin de vendre des magazines.

\section{Conclusion}

42 La présente étude vise à montrer de quelles manières et pour quelles raisons le franglais fait partie intégrante de la presse à scandale française. Notre démonstration s'effectue via l'analyse des lexèmes anglais insérés dans les gros titres, les titres, et les articles des tabloïdes. L'utilisation de l'anglais dans la presse people est, selon nos observations, codée et ciblée, et le nombre de lexèmes anglais qu'elle renferme est conséquent. L'un des objectifs de cette analyse étant également d'identifier les raisons principales de ce phénomène linguistique particulier dans la presse people, nous avons traité les questions d'économie du langage et d'identité de groupe, afin de montrer que ces deux éléments constituent les principales motivations du franglais dans la presse à scandale.

43 À l'avenir, il serait intéressant d'élargir l'étude du lexique anglais à d'autres tabloïdes européens. Si le recours à l'anglais est courant dans la presse people italienne ou allemande, il est en revanche absent de la presse hispanique. Cet axe de recherche nous amènerait à découvrir quelles seraient les spécificités de ce lexique anglais et quelles seraient les raisons de sa présence dans les tabloïdes (économie du langage, identité de groupe, autre ?).

Nous pourrions également envisager de mener une analyse sur le lexique utilisé dans les tabloïdes britanniques et américains, dans le but de relever un ou plusieurs systématismes linguistiques. Concernant l'utilisation d'une langue étrangère, nous 
pourrions, à l'inverse de la présente étude, nous pencher sur le lexique français utilisé dans la presse people anglophone. Si un tel usage était constaté, il s'agirait d'analyser, comme nous l'avons fait au fil de cette contribution, le type de vocabulaire utilisé ainsi que les potentielles motivations telles que la suivante: le français est-il utilisé lorsque l'article est en rapport avec une personnalité française, dans l'objectif de faire correspondre le fond et la forme? Nous nous pencherions aussi sur les valeurs des deux langues en termes de représentation : valeurs culturelle, identitaire, affective, etc.

Il serait également pertinent de se pencher sur le traitement de l'actualité people dans des journaux non spécialisés dans la presse à sensation tels que Le Monde ou Le Figaro. Bien qu'il possède une rubrique intitulée People, Le Monde ne parle pas de "stars" ou de "people» mais privilégie la dénomination "célébrités». Quant au Figaro, la rubrique « actu people » se trouve dans le supplément du samedi Madame Figaro, qui traite de mode, de beauté, de cuisine, donne l'horoscope, etc. Contrairement aux articles du Monde, les dénominations «stars» et "people» sont employées. Le reste du lexique anglais que nous avons étudié au cours de cet article n'apparaît pas non plus dans ce type de presse, ce qui montre qu'il est spécifique des tabloïdes. Plusieurs pistes de recherches pourraient être explorées concernant le traitement de l'actualité people dans la presse d'information. En termes de contenu informationnel, il serait intéressant de se pencher sur ce qui est raconté de la vie privée des stars, dans ce type de presse. Le traitement de leur actualité semble être plus sérieux, plus profond, et plus bienveillant que celui proposé dans les tabloïdes, comme le montre les titres d'articles suivants extraits de Madame Figaro: "Toujours moins bien payée qu'un homme, Julianne Moore défend l'égalité des salaires "; "Sophie Turner s'engage à boycotter les États américains où l'IVG est restreinte »; "Taylor Swift ne répond plus aux questions sexistes »; " Ellen DeGeneres révèle avoir été sexuellement agressée par son beau-père à l'adolescence ». En termes de perception, il serait pertinent d'analyser ce que le lectorat de ce type de presse ainsi que ses journalistes pensent des rubriques people. Sont-elles aussi négativement perçues que celles des tabloïdes? Nous pourrions également nous interroger sur la place qui est octroyée aux informations relatives aux célébrités dans ce type de presse. Ces journaux acceptent de traiter ce type d'actualité, mais l'assument-ils ? La catégorie People du Monde n'est pas très fournie et Le Figaro relaye les informations people dans sa version féminine hebdomadaire Madame Figaro, qui propose également des recettes de cuisine, des astuces beauté, etc. Que pouvons-nous tirer comme conclusions de la place que ces journaux accordent aux people? Traitent-ils uniquement par défaut de l'actualité des célébrités car elle fait vendre (Labarthe 2009) ? Étant reléguée à la version féminine du Figaro, pourquoi l'actualité people serait-elle davantage destinée à un public féminin plutôt que masculin ?

Enfin, le lexique utilisé dans d'autres domaines de la presse écrite "de spécialité ", à savoir les magazines féminins de décoration et de rénovation d'intérieur, par exemple, constituerait également un sujet d'étude pertinent. En effet, dans le but de donner des conseils de décoration et de rénovation, les magazines français Elle, Vie Pratique, ou encore $A u$ féminin, font usage d'un vocabulaire teinté d'anglais, suite à la démocratisation de certains concepts tels que le home staging (entre la décoration et la rénovation), le home relooking (le relooking d'intérieur), le home design (l'architecture d'intérieur), ainsi que le roof garden (le fait d'avoir un jardin sur le toit), popularisés en France par de nombreuses émissions télévisées, notamment $D \& C$, Maison à vendre, et Redesign: sauvons les meubles! sur M6, La Maison France 5, Téva Déco, ou encore Rénovation impossible sur 6ter. La volonté d'être dans l'air du temps et de relayer un maximum de nouveautés serait-elle synonyme 
d'anglicisation des médias tels que la télévision et la presse écrite française ? Pouvonsnous attribuer cette anglicisation aux traductions et adaptations que font les médias français (télévision, radio, journaux, etc.) des programmes, émissions, et magazines anglo-saxons, comme confessé par les deux chefs d'une rubrique people (Labarthe 2009)? Ce phénomène faisant écho à la notion de "tradition discursive » (Loiseau 2013), il mériterait alors d'être élargi au niveau des médias et ne serait pas uniquement spécifique de la presse people.

\section{BIBLIOGRAPHIE}

ALlaOUA, Mourad. 1992. «L'anglo-américanisation du français ». Communication \& Langages 92, 74-84.

« Amber Heard : elle a un nouveau petit-ami, et ce n'est pas n'importe qui ! ». 2018. Public.fr, consulté le 8 septembre 2018 <https://www.public.fr/News/Amber-Heard-Elle-a-un-nouveaupetit-ami-et-ce-n-est-pas-n-importe-qui-1473095>.

ASHFORTH, Blake E. et MAEL, Fred. 1989. "Social Identity and the Organization". Academy of Management Review 14/1, 20-39.

BALLE, Francis. 1994. Introduction aux médias. Paris : Presses Universitaires de France.

BALLE, Francis. 1998. Dictionnaire des médias. Paris : Larousse.

BARDELOT, Estelle. 1999. « Lire la " presse people » ». Mémoire de DEA, Université Lumière Lyon II, sous la direction de Tétu Jean-François, consulté le 18 mai 2019 <https://www.enssib.fr/ bibliotheque-numerique/documents/1655-lire-la-presse-people.pdf>.

BELLEMIN-NOËL, Jean. 1983. Les contes et leurs fantasmes. Paris : Presses Universitaires de France. BOGAARDS, Paul. 2008. On ne parle pas franglais : la langue française face à l'anglais. Bruxelles : De Boeck Duculot.

BROWN, Penelope \& LEVINSON, Stephen C. 1987. Politeness : Some universals in language usage. Cambridge : Cambridge University Press.

BRULEY, Michel. 2018. « Le franglais, la seule langue que l'ensemble des Français maîtrise ! ». M@batim 12 décembre, consulté le 18 mai 2019

$<$ https://mabatim.info/2018/12/12/le-franglais-la-seule-langue-que-lensemble-des-francaismaitrise/>.

CHÉDOTEL, Frédérique. 2004. « Avoir le sentiment de faire partie d'une équipe : de l'identification à la coopération ».M@n@gement 7/3, 161-193.

CULPEPER, Jonathan. 2011. Impoliteness : Using Language to Cause Offense. Cambridge : Cambridge University Press.

DELPORTE, Christian. 2006. « Les recettes de la presse people ». L'Histoire janvier, consulté le 18 mai 2019 <https://www.lhistoire.fr/les-recettes-de-la-presse-people>. 
DUCAS, Marie-Claude. 2014. « Franglais? ? Vous devriez voir en France ! ». Le Journal de Montréal 28 juillet, consulté le 18 mai 2019

<https://www.journaldemontreal.com/2014/07/28/franglais-vous-devriez-voir-en-France>. DUNBAR, Giselle. 2017. « Anglicismes : sont-ils utilisés pour la praticité ou parce qu'ils sont à la mode? », Veille du Centre de Formation des Traducteurs, Terminologues et Rédacteurs techniques CFTTR, consulté le 31 mai 2019

$<$ https://www.sites.univ-rennes2.fr/lea/cfttr/veille/2017/03/09/anglicismes-sont-ils-utilisespour-la-praticite-ou-parce-quils-sont-a-la-mode/>.

EL HIMANI, Rime. 2017. « Photo - Céline Dion retrouve Pepe Munoz pour un show érotique ». Gala.fr 29 novembre, consulté le 8 septembre 2018

$<$ https://www.gala.fr/1_actu/news_de_stars/photo-celine-dion-retrouve-pepe-munoz-pour-unshow-erotique_409803>.

ESQUENAZI, Jean-Pierre. 2003. « Voici et le star-system ». Médiamorphoses 8, 61-67.

ÉTIEMBLE, René. 1973. Parlez-vous franglais ?. Paris : Gallimard.

FAURE, Mélanie. 2018. « Vanessa Paradis pousse un coup de gueule sur sa love story avec Samuel Benchetrit : « Je n'ai pas à le partager » ». Closer.fr, 16 novembre, consulté le 20 mai 2019

$<$ https://www.closermag.fr/people/vanessa-paradis-pousse-un-coup-de-gueule-sur-sa-lovestory-avec-samuel-benchetri-899670>.

Gala. Consulté le 8 septembre 2018 <https://www.gala.fr>.

HAUGEN, Einer. 1977. "Norm and Deviation in Bilingual Communities”, in P. Hornby, Bilingualism : Psychological, Social and Educational Implications. New York : Academic Press, 91-102.

HOFFMAN, Charlotte. 1991. An Introduction to Bilingualism. London : Longman.

« Ian Somerhalder et sa girlfriend Nina Dobrev, très glamour, arrivent à l'aéroport de LAX pour se rendre à Atlanta ». 2018. Purepeople.fr, consulté le 20 mai 2019

<http://www.purepeople.com/media/ian-somerhalder-et-sa-girlfriend-nina_m676584>. JILOT-GUÉRAND, Anne-Sophie. 2018. « Photos : Alizée topless à la plage... attention, c'est chaud !». Public.fr 30 juillet, consulté le 8 septembre 2018 <https://www.public.fr/Toutes-les-photos/ Photos-Alizee-topless-a-la-plage-attention-c-est-chaud-1483482>.

JILOT-GUÉRAND, Anne-Sophie. 2018. « Zazie topless, la photo osée dont TOUT le monde parle ! ». Public.fr 13 juillet, consulté le 8 septembre 2018

<https://www.public.fr/News/Zazie-topless-la-photo-osee-dont-TOUT-le-monde-parle-1480305>. JOUVE, Vincent. 1992. L'effet-personnage dans le roman. Paris : Presses Universitaires de France. « La presse people rend-elle con?». 2011. L'obs, Le Plus 22 septembre, consulté le 18 mai 2019 <http://leplus.nouvelobs.com/contribution/195119-la-presse-people-rend-t-elle-con.html>. LABARTHE, Gilles. 2009. « L'extension du people dans la presse quotidienne ». Communication 27/1, 220-236.

LACAN, Jacques. 2006. Le séminaire XVI : d'un autre à l'Autre. Paris : Seuil.

LALANDE, André. 1968. Vocabulaire technique et critique de la philosophie. Paris : Presses Universitaires de France. 
LANDRAGIN, Frédéric. 2006. «Saillance ». Sémanticlopédie : dictionnaire de sémantique, consulté le 26 décembre 2018 <http://www.semantique-gdr.net/dico/index.php/Saillance>.

«Le royal wedding a 2 mois : 30 photos pour revivre le conte de fées de Meghan et Harry! ». 2018. Public.fr, consulté le 8 septembre 2018 <https://www.public.fr/Toutes-les-photos/Le-royalwedding-a-2-mois-30-photos-pour-revivre-le-conte-de-fees-de-Meghan-et-Harry-1481803>.

LITS, Marc. 2009. « La construction du personnage dans la presse people ». Communication 27/1, 124-138.

LOISEAU, Sylvain. 2013. «La notion de tradition discursive : une perspective diachronique sur les genres textuels et sur les phenomenes de frequence textuelle ». Pratiques 157-158, 91-104.

«M. Pokora exilé à Los Angeles : sa paisible love life avec Christina Milian ». 2018. Purepeople.fr, consulté le 20 mai 2019 <http://www.purepeople.com/article/m-pokora-exile-a-los-angeles-sapaisible-love-life-avec-christina-milian_a270406/1>.

MARION, Philippe. 1999. « Les mirages de la révélation promise. Le discours de la presse à sensation ». Médiatiques 16, 6-10.

MARTINET, André. 1955. Économie des changements phonétiques. Berne : Francke.

MARTINET, André. 1980. Éléments de linguistique générale. Paris : Armand Collin.

MATHIEU, Clément. 2018. «Pourquoi le royal baby s'appelle-t-il Louis, Arthur, Charles?». ParisMatch.com 3 mai, consulté le 8 septembre 2018 <https://www.parismatch.com/Royal-Blog/ Royaume-Uni/Pourquoi-le-royal-baby-s-appelle-Louis-Arthur-Charles-1508510>.

MORIN, Edgar. 1984. Sociologie. Paris : Fayard.

MORIN, Edgar. 2008. L'esprit du temps. Paris : Armand Colin.

« Oui, je parle anglais. Et alors?». 2018. Heureux comme Ulysse, consulté le 18 mai $2019<$ https:// heureuxcommeulysse.com/parler-franglais-et-alors/>.

«Pamela Anderson et Adil Rami : alerte à la love story ». 2018. Purepeople.fr, consulté le 20 mai 2019 <http://www.purepeople.com/article/pamela-anderson-et-adil-rami-alerte-a-la-lovestory_a307463/1>.

Paris Match. Consulté le 8 septembre 2018 <https://www.parismatch.com>.

«Photos : Ariana Grande : déjà un nouveau boyfriend?».2018. Public.fr, consulté le 8 septembre 2018

<https://www.public.fr/Toutes-les-photos/Photos-Ariana-Grande-Deja-un-nouveauboyfriend-1472591>.

Public. Consulté le 8 septembre $2018<$ https://www.public.fr>.

Purepeople. Consulté le 8 septembre 2018 <http://www.purepeople.com>.

RAIS, Cécile. 2005. L'événement people. Quelle subjectivité pour quelle presse. Mémoire, sous la direction de Antoine Maurice, Université de Neuchâtel.

ROUYER, Marion. 2018. " Le royal baby 3 le fils de Kate Middleton et William rapporte déjà gros ». Gala.fr 4 mai, consulté le 8 septembre 2018

$<$ https://www.gala.fr/l_actu/news_de_stars/le-royal-baby-3-le-fils-de-kate-middleton-etwilliam-rapporte-deja-gros_414971>. 
ROWLETT, Paul. 2006. "Franglais", in K. Brown \& S. Ogilvie, Concise Encyclopedia of Languages of the World. Oxford : Elsevier Ltd., 425-426.

SALL, Elisabeth. 2018. « Drake n'est plus célibataire ! Découvrez le visage de sa nouvelle girlfriend ». Public.fr 17 mai, consulté le 8 septembre 2018

<https://www.public.fr/News/Drake-n-est-plus-celibataire-Decouvrez-le-visage-de-sa-nouvellegirlfriend-1471902>.

SANTROT, Florence. 2018. « Royal wedding : Meghan Markle et le Prince Harry faits duc et duchesse de Sussex ». Grazia.fr 19 mai, consulté le 8 septembre 2018

$<$ https://www.grazia.fr/people/royal-wedding-meghan-markle-et-le-prince-harry-faitsduc-etduchesse-de-sussex-888650>.

SCHICKEL, Richard. 1985. Intimate strangers : the culture of celebrity. New York : Doubleday.

SWALES, John M. 1990. Genre analysis. English in academic and research settings. Cambridge :

Cambridge University Press.

TISSERON, Serge. 2001. L'intimité surexposée. Paris : Ramsay.

TISSERON, Serge. 2011. « Intimité et extimité ». Communications 88, 83-91.

« Torride, Bella Hadid pose topless en couverture de Vogue ». 2018. ParisMatch.com, consulté le 8 septembre $2018<$ https://www.parismatch.com/People/Torride-Bella-Hadid-pose-topless-encouverture-de-Vogue-1550120>.

TOURNIER, Michel. 2002. Journal extime. Paris : Gallimard.

VINCENT, Coralie. 2018. «Sam Asghari métamorphosé : le boyfriend ultra-sexy de Britney Spears ne ressemblait pas DU TOUT à ça ». Closer.fr 19 mars, consulté le 9 septembre 2018 <https:// www.closermag.fr/people/sam-asghari-metamorphose-le-boyfriend-ultra-sexy-de-britneyspears-ne-ressemblai-791714>.

Voici. Consulté le 8 septembre 2018 <https://www.voici.fr>.

\section{ANNEXES}

Annexe 1 - Captures d'écran des magazines français

Gala, Paris Match, Public, Purepeople, et Voici 
Paris Match

hitps://WwW.parismatch.com/ - Translate this page

Découvrez le site de Paris Match ! Tous les jours, retrou

lactualité de vos people préférés mais également les..

\section{People}

Suivez les actualités de vos People

préferéés. Découvrez les.

Actualités

Toutes les actualités nationales et

internationales sont sur Paris.

Le Roval Blog

Monaco - Culture - Famille royale de

Suède - Famille royale belge

Actu people et stars - Public.fr

https $/ /$ www.public.fir * Translate this page

Public.fr : Suivez toute lactualité people en continu I Photos et vidéos, stars françaises et américaines,

buzz du moment, mais aussi mode, beauté et pop culture, ...

News People

Suivez toute Pactualité des collébrités :

news pecole, photos ..

News

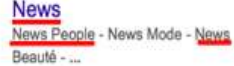

People

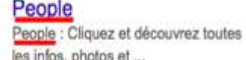

Les scoops Public sur votre ...

Vous allez adorere lapplil I Toules ites

nams Public sur votre mobile.

News TV

Vidé : Zapping TV du 7 septembre:

Quand Kelly ...

\section{VDM People}

VDM People: Cliquez et décowre:

Mode

Mode : Cliquez et découvrez toutes

les infos, photos et ...

Vidéos

Cliquez et découvrez en temps réel

toutes les vidéos peoplo.

Horoscope

Betier - Vierge - Lion - Taureau -

Look de stars

lookde stars: Cliquez et découvrez
Purepeople: People - news people et actu

ww.purepeople.com - Transitete this page

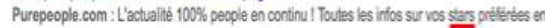

france et at tétranger avec des photos et des vidéos...

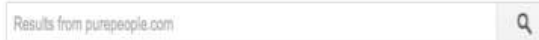

Livenews RSS

Retrowvez toutes les news people $\quad$ Un flux RSS (realy Simple

avec le Livenews de... Sndicaton) permet de recencit-

People france Famille royale

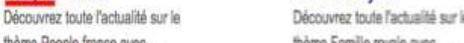

thème Peonde france avec.._. theme Fanlle rojele anc.

Stars: toute l'actu des stars - Gala

hitps://mw. gala.frl $~+$ Transiate this page

Vivez au rythme des stars avec Galatr. Leur actualte, leurs biographies, leurs secrets, leur vie au quotidien. Sans oublier les jeux L...

News de stars Magazine

Tonnews News de stars, Diana : $21 \quad$ Loana en cowverture du magazine

ans après sa mort, la lettre .... El. Ele : « C Ca va clouer le bec à un .

Voici

https://Wmw.voici.fr/ - Translate this page

Toutes les news people sont sur Voici I decoouvrez des articles, diapos et vidéos sur toutes vos stars

Actu people

L'actu people Compte promo ?

shnny Hallyday : son compte...

News People

L'actu peogle. Hantí par ses vieux

Con rest pas du Johnny . VIDEO

Bermard Montiel deflend Laura.

Actualités Anne Gravoin

Anne Gravoin : sa maniêre de
surmonter sa rupture avec...

\section{NOTES}

1. Dans son mémoire de recherche, Bardelot (1999: 44) a recueilli les témoignages de plusieurs lectrices de presse people qui lui ont permis de découvrir l'existence d'un «décalage entre les pratiques culturelles effectives et les discours tenus sur ces mêmes pratiques. Il existe ainsi une population de lecteurs de «presse people » qui dénigre leur lecture en public : ils seront nommés «les consommateurs non-avoués » ».

2. Nous parlerons volontairement de "franglais » et non d' " anglicisme », dans cette étude, car ce dernier n'englobe pas la notion de « quantité » attribuée au franglais.

3. Par le biais de son article «Oui, je parle franglais. Et alors?» (2018), l'auteur du blog intitulé «Heureux Comme Ulysse : pour les parents expatriés et leurs heureux enfants!» aborde la mauvaise perception du franglais qu'ont de nombreux Français et justifie l'emploi qu'il ou elle fait de l'anglais en français afin d'assurer que ce n'est en aucun cas «pour en mettre plein la vue ", contrairement à ce qui peut lui être reproché.

4. L'analyse menée par Haugen est en lien avec la notion d' "alternance codique » et non de «franglais». Cependant, nous considérons que le franglais se distingue principalement de l'alternance codique en ce sens qu'il n'est pas un terme savant ni académique, mais s'apparente davantage à une vulgarisation des termes «alternance codique» (Rowlett 2006: 425) et «emprunt", selon les situations. Ainsi, nous prenons modèle sur les travaux d'Haugen en matière de perception de l'alternance codique afin de développer la perception du franglais.

5. Extrait et traduit de la citation en anglais suivante : They hold that such infringements on the rhetorical norm, as they conceive it to be, are due either to laziness, a moral defect, or to ignorance, an intellectual defect, or to snobbery, a social defect (Haugen 1977 : 94).

6. Via l'expression "auditoire direct et/ou indirect ", nous renvoyons aux participantees de la conversation ainsi qu'aux personnes exclues de cette conversation mais qui sont, toutefois, suffisamment proches pour l'entendre ou, du moins, en saisir des bribes. 
7. Dans cette citation, le verbe pepper retient particulièrement notre attention car, traduit en français par « saupoudrer en grande quantité » ou encore «truffer », il implique, dans notre cas d'étude, une insertion de mots d'origine étrangère abondante.

8. Nous utiliserons le terme « lexème " pour renvoyer aux termes lexicaux, par opposition aux termes grammaticaux.

9. Dans cette étude, l'italique sera utilisé pour les mots anglais ne figurant pas dans les dictionnaires français.

10. Dans sa rubrique consacrée aux people, Le Monde traite de l'actualité des "célébrités » différemment des tabloïdes et n'utilise pas les emprunts « stars ", " people », boyfriend, girlfriend, topless, etc. De la même manière, Le Figaro a une rubrique people dans son supplément Madame Figaro, mais ne propose pas le même contenu people que les tabloïdes. Ce qui est relaté dans les journaux tels que Le Monde et Madame Figaro concernant les stars ressemble bien moins à des potins que les articles de la presse à sensation. Voir les exemples fournis en conclusion de l'article.

11. Le mot ou l'expression la plus importante de l'énoncé.

12. Nous souhaitons préciser que, de la même manière que nous ne mettons pas au même niveau les lexèmes empruntés "topless » et «buzz », par exemple, et les lexèmes issus de l'alternance codique livenews et royal wedding, nous ne mettons pas non plus au même niveau des lexèmes empruntés tels que "people » et "news » et un lexème emprunté tel que "star ", ancré dans le lexique français comme en attestent les dictionnaires depuis des décennies.

13. Selon nous, l'ensemble de la presse française n'adopte pas cette pratique pour deux raisons principales. Premièrement, comme abordé en introduction, le franglais est bien trop critiqué pour que les journaux d'information français ne prennent le risque de teinter leurs articles de mots anglais. Deuxièmement, par souci de formalité, les journaux français dits sérieux évitent d'alterner le français et l'anglais. La notion de familiarité que nous développons dans la souspartie suivante, par opposition à la notion de «formalité », doit être prise en compte dans l'étude du franglais.

\section{RÉSUMÉS}

Cet article vise à développer de quelles manières et pour quelles raisons le franglais, en règle générale décrié, est utilisé dans les tabloïdes français qui ont également mauvaise presse. Via un corpus constitué de gros titres, titres, et extraits d'articles provenant des magazines en ligne de presse à scandale français Closer, Gala, Grazia, Paris Match, Public, Purepeople, et Voici, nous débutons notre analyse par une première partie consacrée aux spécificités $d u$ franglais appliquées à la presse à sensation française, à savoir l'aspect codé et ciblé de ce lexique ainsi que la notion de «quantité ». Puis, dans une seconde partie, nous traitons des principales raisons justifiant l'utilisation du franglais dans ce type de presse en abordant les notions clés d' « économie du langage » (Martinet 1955) et d' « identité de groupe » (Brown et Levinson 1987).

This article aims to develop how and why Franglais, which is, as a general rule, condemned, is used in French tabloids, which also receive bad reviews. Thanks to a corpus composed of headlines, article titles, and excerpts from articles coming from the online French tabloids Closer, Gala, Grazia, Paris Match, Public, Purepeople, and Voici, we start our analysis with a first part devoted to the specific features of Franglais applied to French tabloids, that is to say the codified 
and targeted aspects of this lexicon as well as the notion of "quantity". Then, in a second part, we deal with the main reasons explaining the use of Franglais in this kind of press by tackling the following key notions : "linguistic economy" (Martinet 1955) and "in-group identity" (Brown and Levinson 1987).

INDEX

Mots-clés : « économie du langage » (Martinet 1955), franglais, « identité de groupe » (Brown et Levinson 1987), lexique, presse à scandale, quantité.

Keywords : Franglais, "in-group identity" (Brown and Levinson 1987), lexicon, "linguistic economy" (Martinet 1955), quantity, tabloids.

\section{AUTEUR}

\section{ELODIE MARTIN}

Université de Lyon, Université Jean Moulin - Lyon 3 (CEL - EA 1663)

Université des Antilles, Pôle Martinique (CRILLASH - EA 4095) 